\title{
On the Emergence of Electric Fields at the Water-C12E6 Surfactant Interface
}

\author{
Rahul Gera ${ }^{\dagger}$, Huib J. Bakker*†, Ricardo Franklin-Mergarejo ${ }^{\ddagger}$, Uriel N. Morzan ${ }^{\ddagger}$, Gabriele Falciani ${ }^{\#}$, Luca \\ Bergamasco $^{\#}$, Indraneel Sen ${ }^{\star}$, Silvia Dante ${ }^{\S}$, Eliodoro Chiavazzo ${ }^{\#}$, and Ali Hassanali*ł \\ ${ }^{\dagger}$ AMOLF, Science Park 104, 1098 XG Amsterdam, The Netherlands. \\ International Centre for Theoretical Physics, Strada Costiera 11 - 34151, Trieste, Italy \\ \#Energy Department, Politecnico di Torino, Corso Duca degli Abruzzi 24, Torino \\ 10129, Italy

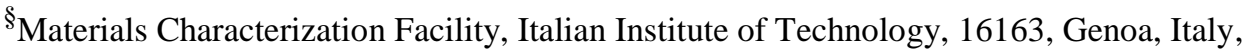 \\ ${ }^{*}$ Uppsala University, Laegerhyddsvaegen 1 - 751 20, Uppsala, Sweden \\ Supporting Information Placeholder
}

\begin{abstract}
We study the properties of the interface of water and the surfactant Hexaethylene Glycol Monododecyl Ether (C12E6) with a combination of Heterodyne-Detected Vibrational Sum Frequency Generation (HD-VSFG), Kelvin-Probe measurements, and Molecular Dynamics (MD) simulations. We observe that the addition of C12E6 close to the critical micelle concentration (CMC), induces a drastic hydrogen bond strength enhancement of the water molecules close to the interface, as well as a flip in their net orientation. The mutual orientation of the water and C12E6 molecules, leads to the emergence of a broad $(\sim 3 \mathrm{~nm})$ interface with a large electric field of $\sim 1 \mathrm{~V} / \mathrm{nm}$, as evidenced by the Kelvin-Probe measurements and MD simulations. Our findings may open the door for the design of novel electric-field tuned catalytic and light harvesting systems anchored at water-surfactant-air interface.
\end{abstract}

\section{INTRODUCTION}

Over the last decades, there has been a significant interest in the behavior of water at inorganic and biological interfaces. ${ }^{1}$ Although it is well appreciated that the structure, dynamics and electronic properties of water change near interfaces, many aspects regarding the spatial extent and magnitude of these perturbations continue to be a topic of intense theoretical and experimental study. ${ }^{2}$ One aspect of specific interest that has recently emerged, is the appearance of electric fields near interfaces and their effect on chemical reactivity. ${ }^{3}$

Electric-field fluctuations are at the heart of chemical reactions such as electron and proton transfer in aqueous solution, in the active sites of proteins, or near DNA. ${ }^{4}$ The role of the electric fields is particularly pertinent when there is a breaking of symmetry in the system, for example at aqueous interfaces due to the presence of ionic charges leading to the formation of a double layer. ${ }^{5}$ Even in the absence of ionic charges, it has been noted that an electric field can be created by a network of oriented water molecules at interfaces. ${ }^{6}$ However, the precise microscopic origin of such electric fields in heterogeneous environments involving water and other solutes, remains elusive, since different species can contribute in non-trivial ways to alter the electrical potentials. The ability to control these electric fields has enormous potential for the development of green-energy technologies. ${ }^{3 \mathrm{~d}, 7}$

In this study we investigate the surface of an aqueous solution of the surfactant Hexaethylene Glycol Monododecyl Ether (C12E6), using a combination of Heterodyne-Detected Vibrational Sum Frequency Generation (HD-VSFG), Kelvin Probe vibrating electrode measurements, and Molecular Dynamics (MD) simulations. C12E6 forms one of the members of a well-studied class of non-ionic surfactants that contain a polyethylene oxide group as the polar head group and an aliphatic chain as the tail. This class of surfactants has been extensively studied over the years for its physical properties. ${ }^{8}$ Moreover, C12E6 is an interesting system as this molecule shows a high potential in the formation of soap films for artificial photosynthesis applications. ${ }^{8 \mathrm{~h}}$

Our findings indicate that C12E6 creates a broad $(\sim 3 \mathrm{~nm})$ interface with strongly enhanced dynamical correlations and hydrogen bonding network involving both co-penetrated water and surfactant groups. The surfactant also induces changes in the water orientation at the interface. These structural changes, observed both by HD-VSFG measurements and MD simulations, create a net interfacial electric field of $\sim 1 \mathrm{~V} / \mathrm{nm}$ pointing towards the bulk which is confirmed by Kelvin probe surface potential experiments. 


\section{MATERIALS AND METHODS}

\section{Experiments:}

\section{Heterodyne-Detected Surface sum-frequency generation (HD-VSFG)}

Surface sensitive VSFG has matured over the years in providing a molecular-level description of interfaces. This technique has been discussed in detail in different reports $^{1 \mathrm{a}, 1 \mathrm{c}, 1 \mathrm{f}, 9}$. In brief, we perform VSFG by focusing two laser pulses with frequencies $\omega_{I R}, \omega_{v i s}$, and on the surface with spatial and temporal overlap. The mid-infrared pulse $\left(\omega_{I R}\right)$ is resonant with the vibrational frequencies of molecules present at the interface, while and $\omega_{v i s}$, is a visible pulse used for up-converting the signal to the sum frequency $\omega_{S F G}\left(\omega_{S F G}\right.$ $\left.=\omega_{I R}+\omega_{V i s}\right)$. Light at $\omega_{S F G}$ can only be created in noncentrosymmetric media under electric dipole approximation, thus making the sum-frequency generation process highly surface specific. The intensity of the sum frequency light created is given by $\mathrm{ISFG}_{\mathrm{SF}} \propto\left|\chi^{(2)}\right|^{2} \mathrm{IVISI}_{\mathrm{IR}} \mathrm{LSFG}^{2} \mathrm{LIR}^{2} \mathrm{LVIS}^{2}$, where $\chi^{(2)}$ is the macroscopic second-order nonlinear susceptibility, IVIS, IIR are the intensities of the incoming visible and mid-infrared radiation respectively, and LsFG, LIR, LvIs is the Fresnel coefficients for the SFG, IR and the visible light respectively. We also measure the VSFG spectrum of a reference sample for which the sum-frequency generation is non-resonant, to normalize the measured signal on the spectral intensity distribution of the infrared pulse. $\chi_{\text {ref }}{ }^{(2)}$ is independent of $\omega_{I R}$ and $\omega$ VIs. Thus using,

$$
\frac{I_{S F G}\left(\omega_{S F G}\right)}{I_{r e f}\left(\omega_{S F G}\right)}=\frac{\left|\chi^{(2)}\left(\omega_{S F G}=\omega_{I R}+\omega_{V i s}\right)\right|^{2}}{\left|\chi_{\text {ref }}^{(2)}\left(\omega_{S F G}=\omega_{I R}+\omega_{V i s}\right)\right|^{2}}
$$

a frequency-dependent $\left|\chi^{(2)}\left(\omega_{S F G}=\omega_{I R}+\omega_{V i s}\right)\right|^{2}$ or the VSFG spectrum is obtained. $\chi^{(2)}\left(\omega_{S F G}=\omega_{I R}+\omega_{V i s}\right)$ can often be expressed as a sum of Lorentzian vibrational resonances and a non-resonant background signal:

$$
\chi^{(2)}\left(\omega_{S F G}=\omega_{I R}+\omega_{V i S}\right)=A_{N R}+\sum_{n} \frac{A_{n}}{\omega_{n}-\omega_{I R}-i \Gamma_{n}}
$$

where $\omega_{n}, A_{n}$, and $\Gamma_{\mathrm{n}}$ are the frequency, amplitude, damping constant of the vibrational mode $\mathrm{n}$ of the molecule at the interface respectively, and $A_{N R}$ is the non-resonant background. Direct determination of $\chi^{(2)}\left(\omega_{S F G}\right)$ from the conventional intensity VSFG is not possible as the intensity VSFG is proportional to $\left|\chi^{(2)}\right|^{2}$, which strongly depends on the interference of the non-resonant background and the resonant contributions to $\chi^{(2)}$. This problem can be solved by

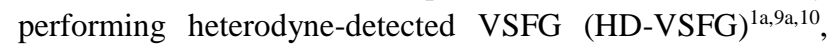
which gives direct access to the real and imaginary part of $\chi^{(2)}$. $\operatorname{Im} \chi^{(2)}$ is a direct representation of the vibrational spectrum of the molecules at the interface and can thus be directly compared to bulk infrared and Raman measurements. ${ }^{\text {1a,9a,10 }}$ Moreover, the sign of $\operatorname{Im} \chi^{(2)}$ reflects the orientation of the molecular group that carries the normal vibrational mode.
The details of the experimental setup have been previously reported. ${ }^{11}$ Briefly, the $\sim 3 \mathrm{~mJ}, 35 \mathrm{fs}, 800 \mathrm{~nm}$ pulses from a regenerative Ti:sapphire amplifier (Coherent Legend) working at $1 \mathrm{kHz}$ repetition rate, are split into two parts. About $2 \mathrm{~mJ}$ of the fundamental beam is used to generate a midinfrared (mid-IR) beam using a tunable home-built optical parametric amplifier (OPA) and a difference-frequency generation (DFG) stage. The $\omega_{I R}$ from the OPA + DFG was centred at $\sim 3000 \mathrm{~nm}$ and the pulses had an energy of $\sim 12-14$ $\mu \mathrm{J}$. Another part of the fundamental beam is sent through an etalon to stretch the femtosecond beam to a few picoseconds and to narrow the frequency bandwidth to $15 \mathrm{~cm}^{-1}$. This beam acts as $\omega_{\text {vis }}$ in HD-VSFG we generate $\omega_{S F G}$ from both a gold substrate that acts as a local oscillator (LO), and from the sample. The two SFG responses are delayed in time with respect to each other by passing the local oscillator $\omega_{S F G}$ beam through a silica plate $(\sim 1 \mathrm{~mm})$. The two $\omega_{S F G}$ beams are sent into a monochromator and detected with an electronmultiplied charge-coupled device (EMCCD, Andor Technologies). The interference pattern of the two beams makes it possible to extract the real and imaginary part of $\chi^{(2)}$ using Fourier transformation. $\operatorname{Im} \chi^{(2)}$ is obtained by comparing the HD-VSFG signal with a reference HD-VSFG signal of which the phase of the SFG light is known. We obtain the reference HD-VSFG by replacing the sample by a z-cut quartz crystal. The typical acquisition time of an HD-VSFG spectrum is $55 \mathrm{~s}$. The measurements reported in this manuscript are recorded with a $\operatorname{ssp}\left(\omega_{S F G}, \omega_{V i s}, \omega_{I R}\right)$ polarization combination.

The stock of C12E6 surfactant ( $\geq 99 \%$ pure) was purchased from Anatrace and used without further purification. We prepare solutions with different surfactant concentrations in millipore water.

\section{Kelvin-Probe Surface Potential}

The Kelvin probe experiments were carried out using a custom-made reduced volume (50 mm x $148 \mathrm{~mm}$ ) Teflon Langmuir trough (KSV-NIMA, Finland). The trough was placed under a hood and equipped with a surface potential sensor with a vibrating electrode (SPOT, KSV-NIMA). The surface pressure was measured in the same trough using a PtWilhelmy probe. The experimental set-up is shown in the SI (Fig. S3).

Before usage, the Teflon trough was carefully cleaned with ethanol and rinsed several times with ultrapure water to remove any trace of contaminants. The SPOT stainless steel counter-electrode was treated in a UV/Ozone chamber for 15 minutes before placing it in the trough. The trough was filled with a measured volume of ultrapure water $(43 \mathrm{~mL})$, and the water surface was carefully cleaned by a vacuum aspirator. The measurements were carried out at $22{ }^{\circ} \mathrm{C}$.

The surface pressure of the pure water was given a reference value of $0 \mathrm{mN} / \mathrm{m}$. The vibrating electrode was placed at $2 \mathrm{~mm}$ 
above the surface of the pure water and a baseline recording was started. After $300 \mathrm{~s}$, a small volume of the surfactant stock solution $(0.04 \mathrm{M})$ was accurately measured and injected at the bottom of the trough using a Hamilton syringe to reach the desired final concentration $(\mathrm{c}=0.07 \mathrm{mM})$. At the same time, a corresponding volume of water was removed from the trough, in order to minimize the change of the water level. The surface potential changes and the surface pressure were continuously recorded for at least 6000s (see Fig. S2).

The C12E6 surfactant (BioXtra $\geq 98.0 \%$ (TLC), SigmaAldrich) was used without further purification. The surfactant was dissolved in ultrapure water (resistivity $18.2 \mathrm{M} \Omega \mathrm{m}$ ) at a concentration $\mathrm{c}=0.04 \mathrm{M}$ and allowed to stabilize for at least $24 \mathrm{~h}$.

\section{Computational Methods}

\section{Molecular Dynamics Simulations}

To study the physical properties of the water-C12E6 mixture in comparison to those of the water-air interface, we performed molecular dynamics (MD) simulations of both systems. Figure 1 depicts the simulated water-C12E6 mixture. The system consists of two interfaces each composed of 48 surfactant molecules close to the critical micelle concentration (CMC). ${ }^{8 \mathrm{e}, 12}$ The cell dimensions of the periodic system are $5 \mathrm{~nm}$ in both the $\mathrm{x}$ and $\mathrm{y}$ directions and $30 \mathrm{~nm}$ in the z-direction. A total of 4055 water molecules were added to build the surfactant-water system illustrated in Figure 1. More details of the simulation can be found in Ref. ${ }^{8 \mathrm{~h}}$.

All the MD simulations were performed using the open source package GROMACS. ${ }^{13}$ In these simulations, we employed the OPLS-AA ${ }^{14}$ force field together with the SPC water model ${ }^{15}$. The combination of interaction potentials were adapted from previous studies ${ }^{8 k, 16}$ which have been shown to reproduce several experimental parameters such as the surface tension and the thickness of the interface ${ }^{8 f, 17}$ For the short-range nonbonded interactions, a cut-off length of $1.0 \mathrm{~nm}$ was chosen for

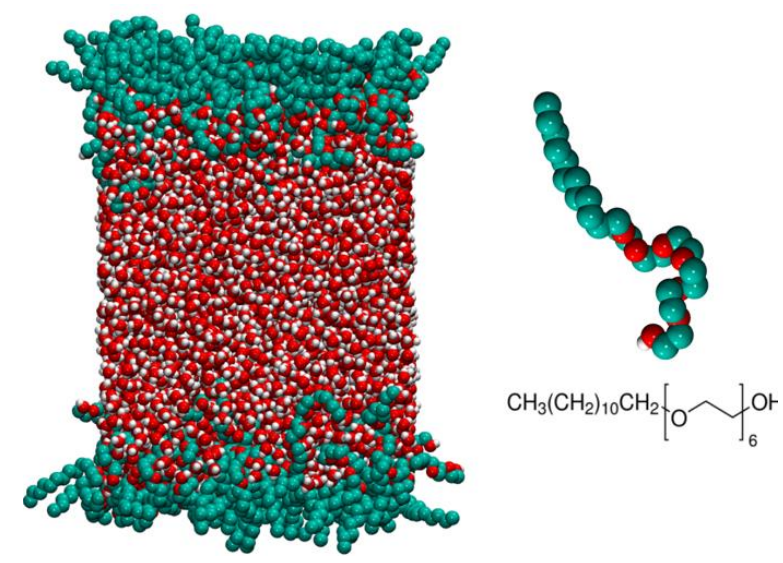

Figure. 1: Representative snapshot of the simulated system. The C12E6 molecules form layers at the interfaces between water and vacuum. Carbon, Oxygen and hydrogen atoms are depicted in green, red, and white respectively. a shifted Lennard-Jones potential while the long-range electrostatic interactions were taken into account via the Particle Mesh Ewald-Switch ${ }^{18}$ (PME-switch) method with a Coulomb switching cut-off of $1.0 \mathrm{~nm}$. A long-range dispersion correction was used to truncate the van der Waals interactions. All bonds were constrained using the LINCS algorithm. ${ }^{19}$ A timestep of 2 fs was used for the Verlet integrator. All simulations were conducted in the canonical ensemble (NVT) at 298.15 K using the Nose-Hoover thermostat $^{20}$ with a time-constant of 2.0 ps. The simulations were carried out for $180 \mathrm{~ns}$ where the first $20 \mathrm{~ns}$ were used for equilibration.

\section{Hydrogen Bond Analysis}

In order to understand how the surfactant changes the water structure, we determined the topology of the hydrogen-bond network. The local water topology is defined by the distribution of the number of hydrogen bonds that are donated (D) and accepted (A) by a water molecule. ${ }^{21}$ Far away from the interface, water molecules will only participate in hydrogen bonds with other water molecules, whereas close to the interface they can form hydrogen bonds both with other water molecules and with surfactant head-groups. The hydrogen bonds were defined using the criterion developed by Luzar and Chandler. ${ }^{22}$

\section{Information Theory Metrics to Study Collective Water- Surfactant Dynamics}

We quantified the correlated motions of water and surfactant molecules with a degree centrality metric (Dc). ${ }^{23}$ The Dc is a score, assigned to every atom in the system, that accounts for the total dynamical correlation between that atom and its neighborhood.

In order to obtain a Dc metric we utilized the linear approximation of the mutual information (MI) introduced by Lange et al. ${ }^{24}$ The mutual information between two particles is defined as:

$$
M I\left[x_{i}, x_{j}\right]=H\left[x_{i}\right]+H\left[x_{j}\right]-H\left[x_{i}, x_{j}\right]
$$

where $x_{i}$ represents the positional fluctuations $\left(x_{i}=\left\langle x_{i}-\underline{x}_{i}\right\rangle\right.$, being $x_{i}$ the three-dimensional position vector), H denotes the entropy of the random variables. The positivity of $M I\left[x_{i}, x_{j}\right]$ rests on the inequality $\mathrm{H}\left[x_{i}, x_{j}\right] \leq \mathrm{H}\left[x_{i}\right]+\mathrm{H}\left[x_{j}\right]$, that becomes an equality if both variables are independent.

The linear mutual information (LMI) relies on a Gaussian approximation of the probability density which can be expressed as:

$$
p\left(x_{i}, x_{j}\right)=\frac{1}{8 \pi^{3} \operatorname{det}\left(\operatorname{Cov}_{i j}\right)}\left(-\frac{1}{2}\left(x_{i}, x_{j}\right) \operatorname{Cov}_{i j}{ }^{-1}\left(x_{i}, x_{j}\right)^{T}\right),
$$


where $\operatorname{Cov}_{i j}=\left\langle\left(x_{i}, x_{j}\right)^{T}\left(x_{i}, x_{j}\right)\right\rangle$ is the covariance matrix, and the entropies $\mathrm{H}$ are evaluated analytically

$$
H\left(x_{i}, x_{j}\right)=\frac{1}{2}\left[q+\ln \left(\left(\operatorname{Cov}_{i j}\right)\right)\right]
$$

where $q=6(1+\ln \ln 2 \pi)=17.02726$. In this study we focus on the correlations that arise from local directional interactions (i.e. hydrogen bonds, dipole-dipole or van der Waals interactions). Hence our estimator accounts for the correlation between each atom and its most immediate neighbors. Therefore, instead of the standard covariance we defined a local covariance $\widetilde{\operatorname{Cov}_{l \jmath}}$ as

$$
\widetilde{\operatorname{Cov}_{l \jmath}}=\left\langle\left(x_{i}, x_{j}\right)^{T}\left(x_{i}, x_{j}\right) \exp -\left[\left(x_{i}-x_{j}\right) / \lambda\right]^{2}\right\rangle \text {. }
$$

The exponential term in equation 4 damps the covariance contribution associated to distant atoms and leaves only the local components with a characteristic distance parameter of $\lambda$. In the present work we employed $\lambda=5 \AA$, which accounts for the first and second solvation shells.

We can now define a local linear mutual information (LLMI) as

$$
\begin{aligned}
\operatorname{LLMI}_{i j}= & {\left[\frac{1}{2}\left(\operatorname{det}\left(\widetilde{\operatorname{Cov}_{l}}\right)\right)+\left(\operatorname{det}\left(\widetilde{\operatorname{Cov}_{J}}\right)\right)-\right.} \\
& \left.\left(\operatorname{det}\left(\widetilde{\operatorname{Cov}_{l J}}\right)\right)\right],
\end{aligned}
$$

where $\widetilde{\operatorname{Cov}_{l}}=\left\langle\left(x_{i}\right)^{T}\left(x_{i}\right)\right\rangle$ represents the marginal covariance. With this definition we can introduce our Dc metric as the total LLMI per neighbor:

$$
D_{c}^{i}=\sum_{j}^{N} \frac{L L M I_{i j}}{\left\langle\exp -\left[\left(x_{i}-x_{j}\right) / \lambda\right]^{2}\right\rangle},
$$

where $D_{c}^{i}$ is the degree centrality for the i-eth atom in the system, indicating the strength of the dynamical "cross-talk" between atom $i$ and its neighbors.

The Dc distribution can be projected on the perpendicular axis to the surfactant plane (z-axis), which enables a determination of the water-surfactant and water-water correlations as a function of the distance to the interface.

In the infinite time limit the Dc distribution becomes uniform due to water diffusion. Therefore, in order to retain the local properties of Dc, we extracted 20 replicas of 3 ps trajectories with $1 \mathrm{fs}$ time step. The resulting z-projected distribution is obtained by averaging the 20 replicas.

\section{RESULTS AND DISCUSSION}

We performed SFG measurements for neat water and solutions with different concentrations of C12E6 in water (see Figure 2 and supporting information (SI), Figure S1). The spectrum of the neat air-water interface shown in Figure 2 consists of a broad band centered at $\sim 3400 \mathrm{~cm}^{-1}$, attributed to the $\mathrm{OH}$ stretch vibrations of water molecules that donate hydrogen bonds to other water molecules, and a narrower band around $3700 \mathrm{~cm}^{-1}$, assigned to the stretch vibrations of non-hydrogen-bonded water $\mathrm{O}-\mathrm{H}$ groups. ${ }^{9 \mathrm{a}, 25}$ The orientation of the water molecules at the interface can be directly obtained from the sign of $\operatorname{Im} \chi^{(2)}$. The $\operatorname{Im} \chi^{(2)}$ of the broad band centered at $\sim 3400 \mathrm{~cm}^{-1}$ has a negative sign, whereas the $\operatorname{Im} \chi^{(2)}$ of the narrower band around $3700 \mathrm{~cm}^{-1}$ is positive. This implies that the water molecules that donate hydrogen bonds to other water molecules have a net orientation of their $\mathrm{O}-\mathrm{H}$ groups towards the bulk of the liquid, whereas the non-hydrogenbond donating $\mathrm{OH}$ groups hydrogen are directed towards the air. $^{9 \mathrm{a}, 25}$

Adding C12E6 to water at concentrations larger than $1 \mu \mathrm{M}$ leads to the disappearance of the band at $\sim 3700 \mathrm{~cm}^{-1}$. This concentration is much lower than the critical micelle concentration (CMC) of C12E6, which is $~ 70-80 \mu \mathrm{M}^{26}$ (See Figure 2 and the Figure S1 in SI, for a detailed comparison of the spectral evolution at different concentrations). The loss of signal at $3700 \mathrm{~cm}^{-1}$ with increasing concentration of C12E6, shows that the surface of water becomes covered by a layer of surfactant molecules. The most striking observation upon adding C12E6 is the change in sign of the $\operatorname{Im} \chi^{(2)}$ of the hydrogen-bonded $\mathrm{OH}$ groups. The negative $\operatorname{Im} \chi^{(2)}$ between
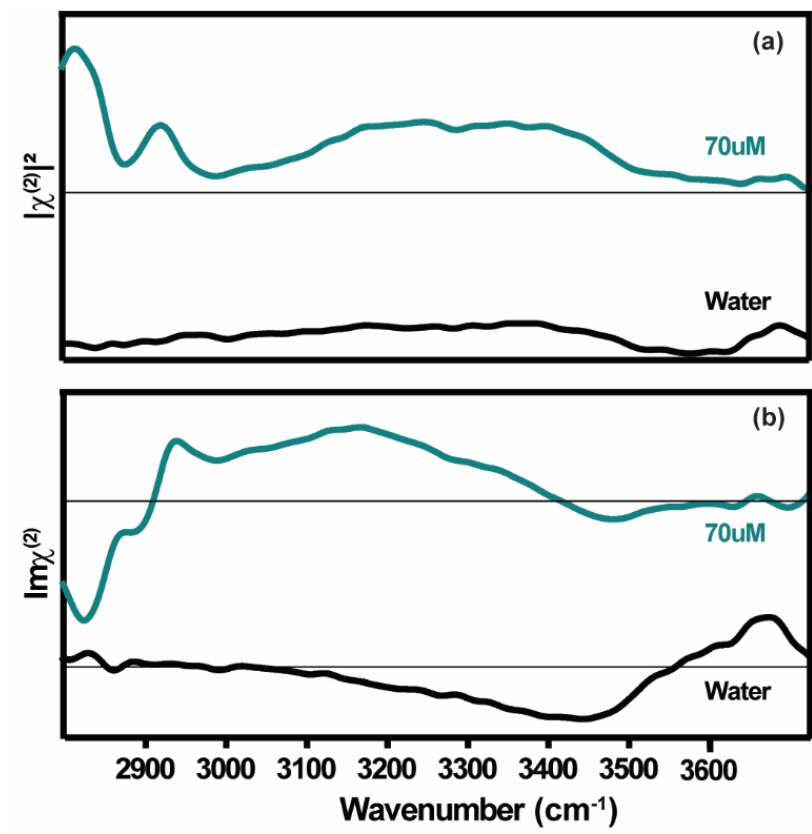

Figure 2. (a) Steady state SFG Intensity spectra of the Air/C12E6/Water interface measured at CMC $\sim 70 \mu \mathrm{M}$ of C12E6 (b) Steady state $\operatorname{Im} \chi^{(2)}$ spectra of Air/C12E6/Water interface measured at $\mathrm{CMC} \sim 70 \mu \mathrm{M}$ of C12E6. 
$\sim 3200$ and $\sim 3600 \mathrm{~cm}^{-1}$ of neat water changes to a strong positive $\operatorname{Im} \chi^{(2)}$ between $\sim 3000$ and $\sim 3400 \mathrm{~cm}^{-1}$. This change of sign for $\operatorname{Im} \chi^{(2)}$ shows that the net orientation of the hydrogen-bonded water molecules at the surface changes from pointing towards the bulk to a net direction towards the surfactants or the air. Adding the surfactant to the solution also leads to a significant red-shift of the response of the hydrogen-bonded $\mathrm{OH}$ groups. More specifically, the broad negative feature $\sim 3400 \mathrm{~cm}^{-1}$ is red-shifted to a more intense positive band centered at $\sim 3200 \mathrm{~cm}^{-1}$. The redshift of the response implies that the hydrogen bonds of the water molecules near the interface have become stronger, indicating that the water molecules form strong hydrogen bonds with the head groups of the surfactants.

In addition to the strong positive band at $\sim 3200 \mathrm{~cm}^{-1}$, the $\operatorname{Im} \chi^{(2)}$ spectrum of the C12E6 solutions also shows a weak negative feature at $\sim 3500 \mathrm{~cm}^{-1}$. In a previous intensity SFG study by Tyrode and co-workers for solutions of C12E4, $\mathrm{C} 12 \mathrm{E} 5$, and C12E8, which are structural analogues of C12E6, the rise of a weak response near $3500 \mathrm{~cm}^{-1}$ was also observed. This response was assigned to weakly hydrogen-bonded water molecules in the tail region of the surfactant molecules. ${ }^{8 \mathrm{~g}, 27} \mathrm{In}$ the same work, the net orientation of the $\mathrm{OH}$ groups of these water molecules was studied by VSFG intensity spectra measured in different polarization conditions, and using information about the hyperpolarizability tensor elements for water based on molecular symmetry. ${ }^{8 \mathrm{~g}, 27}$ Based on this analysis, the authors concluded that the $\mathrm{OH}$ groups of the water molecules with vibrational frequencies $\sim 3500 \mathrm{~cm}^{-1}$ would be oriented towards the tail of the surfactant molecules. i.e. away from bulk water. In the present HD-VSFG measurements, we determine the orientation directly from the sign of $\operatorname{Im} \chi^{(2)}$. We observe that $\operatorname{Im} \chi^{(2)}$ has a negative sign at these frequencies, which unambiguously shows that the weakly hydrogen-bonded $\mathrm{OH}$ groups of water molecules in between the tails of the surfactant molecules have a net orientation towards the head group of the surfactant or the bulk of the solution.

The addition of C12E6 also leads to the rise of signal contributions between $\sim 2800$ and $\sim 3000 \mathrm{~cm}^{-1}$. These signals are attributed to the $\mathrm{CH}$ stretch vibrations of methylene, $-\mathrm{CH}_{2}-$ and methyl groups, $-\mathrm{CH}_{3}$ of the ethylene oxide head group and the alkyl tail group of the surfactant. When the concentration of $\mathrm{C} 12 \mathrm{E} 6$ is increased and approaches the $\mathrm{CMC}$, the response of the $\mathrm{C}-\mathrm{H}$ stretch vibrations of the surfactant molecules in the frequency region $2800-3000 \mathrm{~cm}^{-1}$ increases in comparison to that of the water $\mathrm{O}-\mathrm{H}$ stretch vibrations (see Figure $\mathrm{S} 1$ in SI). This change is a direct consequence of the evolution in the packing of the surfactant at the interface as its concentration is increased: the surface area per molecule at full surface coverage (near CMC) corresponds to approximately $\sim 62 \AA^{2}$ per surfactant molecule. ${ }^{8 a}$ Such tight packing at the interface with increasing concentration increases the relative amplitude of $\mathrm{C}-\mathrm{H}$ stretching region in comparison to $\mathrm{O}-\mathrm{H}$ stretching region of the spectrum, in line with previously reported work with the structurally similar surfactant $\mathrm{C} 12 \mathrm{E} 5^{8 \mathrm{~g}, 27}$

The increasingly tight packing of surfactant molecules with increasing C12E6 concentration is accompanied by a decrease in the amplitude of the $\operatorname{Im} \chi^{(2)}$ response and SFG intensity of the water $\mathrm{OH}$ groups, as shown in Figure S1 of the SI. The tighter packing with increasing surfactant concentration also leads to a narrowing of the negative $\operatorname{Im} \chi^{(2)}$ signal at $\sim 3500 \mathrm{~cm}^{-}$ ${ }^{1}$, as can be seen in Figure S1 in SI. The initial broad signal near $\sim 3500 \mathrm{~cm}^{-1}$ reflects a large variation in hydrogen-bond strength of the water molecules that percolate in the hydrophobic tails. As the concentration of the surfactant is increased, the alkyl tails are more closely packed which induces an ordering of the water molecules, thereby making the feature at $\sim 3500 \mathrm{~cm}^{-1}$ narrower.

To better understand how the mutual polarization of the water and surfactant alters the dielectric properties, we measured the surface potential of the water-C12E6 interface via a vibrating electrode method, or Kelvin probe, according to the working principle described in the supplementary material (see Figure S3). The potential difference is measured between an upper electrode, vibrating vertically at $2 \mathrm{~mm}$ distance above the gasliquid interface, and a fixed counter electrode immersed in the liquid. This technique allows then to characterize changes in the electric potential of the air-water interface induced by surfactant monolayers. The surface potential is defined as:

$$
V_{\text {surf }}=V-V_{0}
$$

where $V$ and $V_{0}$ are the potentials in presence or absence of the surfactant monolayer respectively. ${ }^{31}$ Close to the CMC conditions, we find that the surface potential is $+0.7 \mathrm{~V}$. The positive sign in the surface potential obtained with the Kelvin probe measurements is consistent with previous measurements of non-ionic surfactants. ${ }^{32}$

In order to elucidate the microscopic origin of the spectral features observed in the HD-VSFG experiments and the change of the surface potential observed in the Kelvin-Probe measurements, we performed molecular dynamics simulations. At the interface, water molecules can form hydrogen bonds with other waters as well as surfactant molecules. In particular, the hydrophilic parts of surfactant have ether oxygens (see Figure 1) that can accept hydrogen bonds from water molecules. We thus examined the concentration of some of the hydrogen bonding patterns of water molecules, as shown in Figure $3 \mathrm{a}$. The 0 on the $\mathrm{z}$ axis corresponds to the Gibbs dividing interface (GDI), i.e. the position along the interface where the density of water is half that of the bulk. The labels in the Figure correspond to different types of water molecules. For example, $\mathrm{I}_{2} \mathrm{O}_{2}$ 

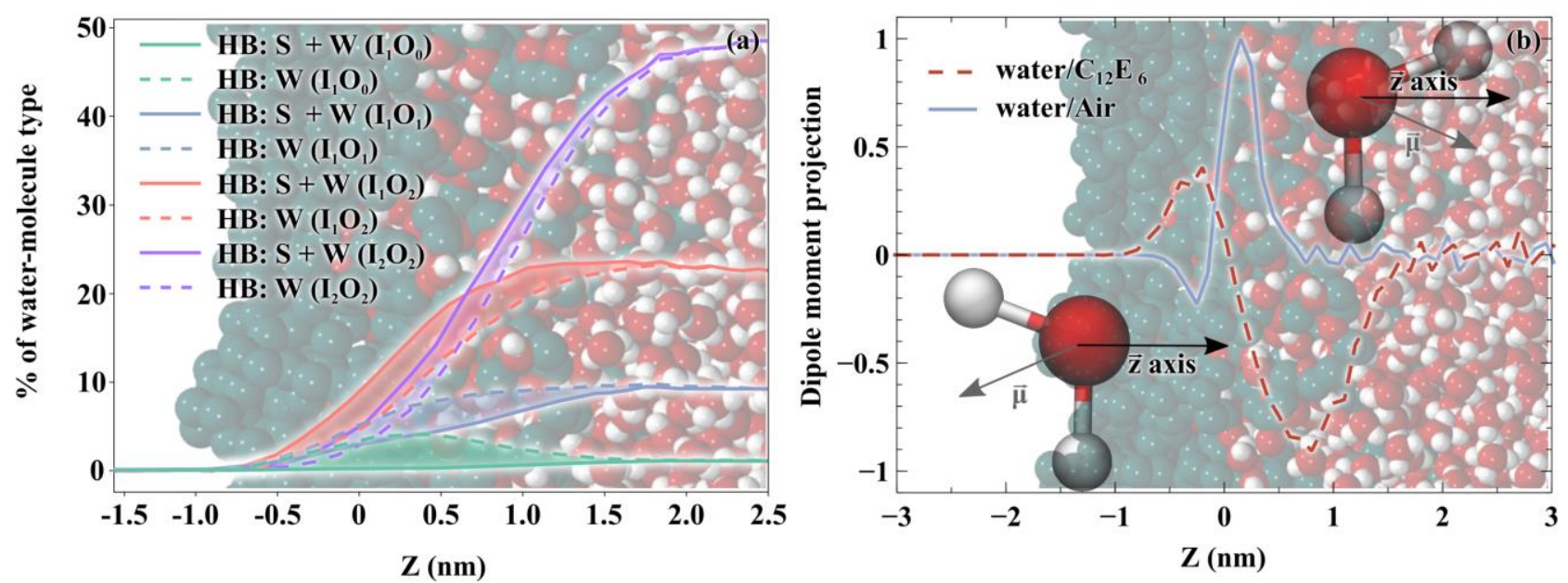

Figure 3: (a) Density of the different types of hydrogen-bonded water molecules by including surfactant groups as possible acceptors/donors of hydrogen bonds. (b) Projection of the total dipole moment of the water molecules on the $\mathrm{z}$ axis as a function of the distance $\mathrm{z}$ from the GDI. The blue curve corresponds to the water at air-water interface and the red dashed curve to the water at the C12E6-water interface. The positive sign indicates a net dipole orientation towards the air. corresponds to water molecules that accept and donate two hydrogen bonds. The $\mathrm{S}$ and $\mathrm{W}$ labels correspond to hydrogen bonding patterns determined with either only water molecules or including both water (W) and surfactant (S).

Interestingly, we observe that the surfactant head-groups act as hydrogen bond acceptors, increasing the local concentration of $\mathrm{I}_{2} \mathrm{O}_{2}$ and $\mathrm{I}_{1} \mathrm{O}_{2}$ (Figure 3a). This corresponds to a decrease in non-hydrogen-bonded (dangling) O-H's since these water molecules now donate hydrogen bonds to the ether oxygens of the surfactant. The relative enhancement in the interfacial population of water molecules that donate and accept two hydrogen bonds, points at an overall strengthening of the hydrogen bond network, in agreement with the red shift observed in the SFG spectra (Figure 2). Interestingly, previous studies of the air-water interface have shown that there is a very thin layer of less than $0.5 \mathrm{~nm}$ that exhibits different hydrogen bonding structures than the bulk. ${ }^{1 \mathrm{a}, 9 \mathrm{a}, 28}$ In contrast, at the air-surfactant-water interface, the changes occur over a rather large length scale of approximately $3 \mathrm{~nm}$.

In Figure $3 b$ it is seen that the water molecules just below the surface acquire a strong orientation with their $\mathrm{OH}$ groups pointing towards the surface, in excellent agreement with the observation of a strong positive $\operatorname{Im} \chi^{(2)}$ centered at $3200 \mathrm{~cm}^{-1}$ in Figure $2 \mathrm{~b}$. The water molecules that are in the hydrophobic region of the surfactant tails have a net orientation towards the bulk, which is again fully consistent with the observation of a negative $\operatorname{Im} \chi^{(2)}$ near $3500 \mathrm{~cm}^{-1}$ in Figure $2 \mathrm{~b}$.

To understand better the collective dynamical perturbation of the hydrogen bond network induced by the presence of C12E6, we turn next to employing an information theory metric. The dynamical behavior of the water molecules is significantly altered in the interfacial region. We

demonstrated this by employing a degree centrality indicator (Dc) as introduced in the Methods section. The $D_{c}^{i}$ is a score associated to the degree of correlation between the motion of the $i$-th molecule and its local environment. The more synchronized these motions are, the higher the $D_{c}^{i}$ value becomes. In this analysis, two types of $D c$ distributions were employed: (i) considering only water motions ( $D c$-wat), and (ii) including both waters and surfactant molecules ( $D c$-tot).

Figure 4 a shows that the $D c$-wat values increase considerably at the interface with respect to the bulk. This indicates that the intrinsic water-water correlations are enhanced at the watersurfactant interface, which is consistent with the strengthening of the hydrogen bond network discussed earlier. Furthermore, the interfacial increase of the $D c$-tot distribution is much more pronounced than that of the Dc-wat values (as seen in the zoom-in plot of Figure $4 \mathrm{~b}$ ). This means that the dynamical correlations between water and head groups are much stronger than those observed between water molecules. These results demonstrate that the strong water-surfactant interactions alter the intrinsic dynamics of water molecules at the interface. The interaction with the head groups thus amplifies the waterwater correlated motions. This dynamically correlated hydrogen-bond network of both water and surfactant leads to non-trivial changes in the dielectric properties as suggested by the Kelvin-probe experiments. To understand the microscopic origins of these changes we examined various dielectric related properties from the atomistic simulations.

Figure 5a shows the total charge density profile at the waterC12E6 surfactant interface (solid black line) as well as the separate charge contributions coming from water (solid blue) and surfactant (red). The specific water orientations and local dipoles of the surfactant, conspire together to yield several 

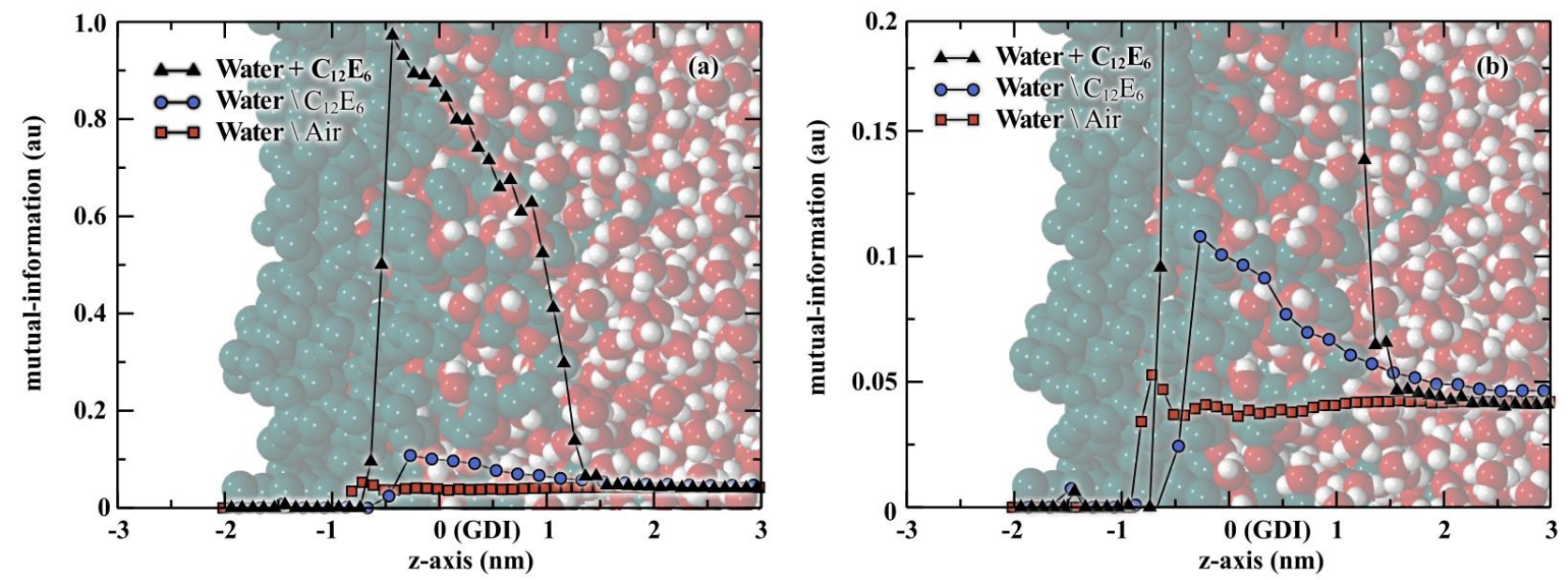

Figure 4: (a) Total z-projection of the $\mathrm{D}_{\mathrm{c}}$ distribution for the water- $\mathrm{C}_{12} \mathrm{E}_{6}$ system (black triangles), for the waters in the presence of the $\mathrm{C}_{12} \mathrm{E}_{6}$ (blue circles), and for the waters in a water-air interface (red squares). (b) Amplification of the right panel. The background depicts the water-surfactant interface. Oxygen, Hydrogen and Carbon atoms are depicted in red, white and green respectively.

layers of alternating positive and negative charges over a length scale of approximately 3 nanometers.

The origin of the modulated charge distribution at the waterC12E6 interface can be well understood by considering the separate contributions of the surfactant and the water molecules, as illustrated in Figure 5a. The interfacial charge distribution consists of two adjacent charge bilayers: (i) a major charge bilayer (Figure 5.a and 5.c, upper positive and negative layers) originated from the polarization between head groups and hydrophobic tails, and (ii) a minor (more subtle) charge bilayer (Figure 5.a and 5.c, lower positive and negative layers) arising from the interplay between water and head-groups relative orientation. The interfacial water molecules that are embedded by the hydrophobic tails have their $\mathrm{OH}$ groups directed towards the bulk, while the much more abundant hydrogen-bonded water molecules deeper down have their $\mathrm{O}-\mathrm{H}$ groups pointing towards the interface, in particular towards the ether oxygen atoms of the surfactant head group. The net result of this charge distribution is a clear charge separation with the negative charge being located closer to the bulk than the positive charge. The molecular origins of these features are schematically summarized in the cartoon of Figure 5c.

The neat water-air interface features only a double layer of charge, as shown in Figure 5d. The upper positive charge is due to the dangling hydrogens from the disrupted hydrogenbond network right at the interface. The oxygen atoms of these water molecules give rise to an excess negative charge that is closer to the bulk. The hydrogen-bonded water molecules have a net orientation that opposes this charge distribution, but this constitutes only a minor contribution.
As a consequence of the charge density distributions described above, an electrostatic potential arises across the interface. We determined the net total electric field, as well as the individual water and surfactant contributions (Figure 5b). The water-C12E6 interface is characterized by an electric field of $\sim 1 \mathrm{~V} / \mathrm{nm}$. Interestingly, the electric field at the airwater interface shown in Figure 5e has a similar magnitude but extends over a much shorter length scale of less than 0.5 nm.

While the values of electric fields at the water-surfactant and air-water interfaces are similar, the origin of the electric fields in the two systems is very different. The change of the sign of $\operatorname{Im} \chi^{(2)}$ in Figure $2 \mathrm{~b}$ thus does not directly report on the magnitude of the total surface electric field but on the contribution of the hydrogen-bonded water molecules to this field. For the water-surfactant system this contribution dominates, while for neat water-air the surface electric field is dominated by the dangling $\mathrm{OH}$ groups (see Figure $5 \mathrm{c}$ and $\mathrm{f}$ ).

The surface potential measured by Kelvin probe experiments can be directly compared with the electric potential, $V_{M D}$, determined from molecular dynamics simulations by integrating the electric field reported in Fig. 4. The electric potentials obtained from the MD simulations are $V_{M D}^{\text {wat-surf }}=$ $1.15[V]$ and $V_{M D}^{\text {wat-air }}=0.62[\mathrm{~V}]$ for the water-surfactant and water-air interfaces respectively. Therefore, we can estimate the surface potential with the MD simulation values, yielding: $\quad V_{\text {surf } M D}=V_{M D}^{\text {wat-surf }}-V_{M D}^{\text {wat-air }}=0.53[\mathrm{~V}]$, which is in good agreement with the Kelvin probe measurements $(0.7[\mathrm{~V}])$.

\section{PERSPECTIVES AND CONCLUSIONS}


In this work, we have combined sum-frequency generation vibrational spectroscopy, Kelvin-probe surface potential measurements and molecular dynamics simulations to study the structural and dielectric properties of the C12E6surfactant interface. This surfactant is currently being prototyped for use in soap bubbles in applications for artificial photosynthesis [https://cordis.europa.eu/project/id/828838] [https://sofiaproject.eu/].

We find that, close to the CMC, this non-ionic surfactant creates an enhanced dynamically correlated hydrogen bonding network, involving both penetrated water and surfactant groups. The surfactant also induces changes in the water orientations at the interface. These structural and orientational changes observed in the SFG are fully consistent with our MD simulations.

Surface potentials and electric fields have been discussed in both experimental and simulation contexts of various interfaces. ${ }^{16,29}$ Very recently, there has been a growing interest in the use of electric-fields to enhance catalytic processes in different types of chemical reactions. ${ }^{3 \mathrm{~d}, 7,33}$ At the C12E6water interface, we show that the structured hydrogen-bond network creates a region on the nanometer length scale with a net electric field of the order of $1 \mathrm{~V} / \mathrm{nm}$ pointing from the vacuum to the bulk. These predictions from the simulations are subsequently confirmed by comparing with surface potential Kelvin-probe experiments. Even though the

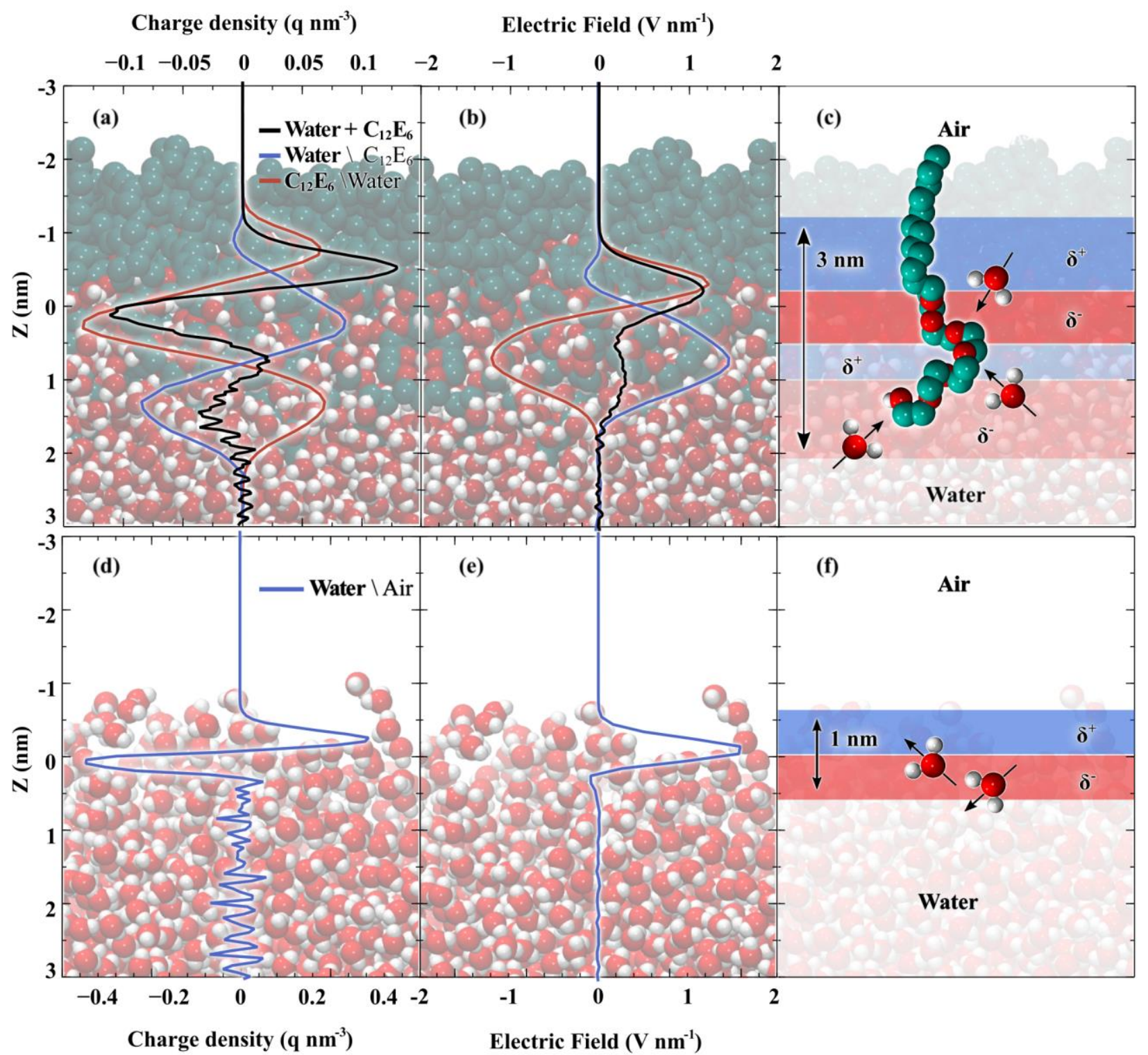

Figure 5. Top panels (a-c) show the charge density (a) and electric fields (b) obtained for the surfactant-water interface. Black curves refer to the total charge density/fields, while the blue and red curves are the individual contributions coming from the water and surfactant respectively. The bottom panels (d-f) correspond to the charge density and electric field arising at the air-water interface. Panels (c) and (f) illustrate the molecular arrangement responsible for the ensuing charge and electric field distributions. 
magnitude of the fields is similar at the water-air and waterC12E6-air interfaces, the changes occur over different length scales $(0.5 \mathrm{~nm}$ vs $3 \mathrm{~nm})$ leading to a big difference in the magnitude of the surface potential.

One of the commonly employed models to interpret the surface potential from Kelvin-probe experiments, considers a dipole field created by the surfactant molecules that is embedded in a continuum dielectric of solvent medium. ${ }^{34}$ This model indicates that the interfacial water within the surfactant has a much smaller dielectric constant than bulk water. Similar features have been observed for water under confinement. ${ }^{35}$ The reduction of the dielectric constant of water at the interface, and the electric fields in this system arising from both the water and the surfactant molecules, have enormous implications for their use in applications such as artificial photosynthesis or catalysis in general. More specifically, a photocatalytic molecule embedded in the surfactant will experience electric fields of varying magnitudes and directions depending on the extent of water or surfactant that surrounds it. Preliminary work from our group shows that electric fields like those observed in the C12E6-water system, can change the HOMO-LUMO gap in Ruthenium-based photocatalysts by at least half an eV. These effects are expected to play a key role in chemical reactions associated with $\mathrm{CO}_{2}$ reduction and water oxidation in artificial photosynthesis applications.

\section{ASSOCIATED CONTENT}

Additional data and information is provided. This material is available free of charge via the Internet at http://pubs.acs.org.

\section{AUTHOR INFORMATION}

\section{Corresponding Author}

* Email: ahassana@ictp.it, $\underline{\text { H.Bakker@ amolf.nl }}$

\section{Funding Sources}

This work has been funded by the European Commission in the framework of the project SoFiA - Soap Film based Artificial Photosynthesis - grant agreement 828838 .

\section{Notes}

Any additional relevant notes should be placed here.

\section{ACKNOWLEDGMENT}

RG would like to acknowledge Carolyn Moll and Jan Versluis from AMOLF for their input while analysis of SFG data and helping with the experimental setup.

\section{ABBREVIATIONS}

Heterodyne-Detected Vibrational Sum Frequency Generation (HD-VSFG), Molecular Dynamics (MD), Hexaethylene Glycol Monododecyl Ether (C12E6), critical micelle concentration (CMC)

\section{REFERENCES}

(1) (a)Nihonyanagi, S.; Yamaguchi, S.; Tahara, T. Ultrafast Dynamics at Water Interfaces Studied by Vibrational Sum Frequency Generation Spectroscopy Chemical Reviews 2017, 117, 10665. (b)Laage, D.; Elsaesser, T.; Hynes, J. T. Water Dynamics in the Hydration Shells of Biomolecules Chemical Reviews 2017, 117, 10694. (c)Jubb, A. M.; Hua, W.; Allen, H. C. Environmental Chemistry at Vapor/Water Interfaces: Insights from Vibrational Sum Frequency Generation Spectroscopy Annual Review of Physical Chemistry 2012, 63, 107. (d)Björneholm, O.; Hansen, M. H.; Hodgson, A.; Liu, L.-M.; Limmer, D. T.; Michaelides, A.; Pedevilla, P.; Rossmeisl, J.; Shen, H.; Tocci, G.; Tyrode, E.; Walz, M.-M.; Werner, J.; Bluhm, H. Water at Interfaces Chemical Reviews 2016, 116, 7698. (e)Park, J. Y.; Baker, L. R.; Somorjai, G. A. Role of Hot Electrons and Metal-Oxide Interfaces in Surface Chemistry and Catalytic Reactions Chemical Reviews 2015, 115, 2781. (f)Geiger, F. M. Second Harmonic Generation, Sum Frequency Generation, and $\chi(3)$ : Dissecting Environmental Interfaces with a Nonlinear Optical Swiss Army Knife Annual Review of Physical Chemistry 2009, 60, 61.

(2) (a)Johnson, C. M.; Baldelli, S. Vibrational Sum Frequency Spectroscopy Studies of the Influence of Solutes and Phospholipids at Vapor/Water Interfaces Relevant to Biological and Environmental Systems Chemical Reviews 2014, 114, 8416. (b)Pettersson, L. G. M.; Henchman, R. H.; Nilsson, A. WaterThe Most Anomalous Liquid Chemical Reviews 2016, 116, 7459. (c)Bellissent-Funel, M.-C.; Hassanali, A.; Havenith, M.; Henchman, R.; Pohl, P.; Sterpone, F.; van der Spoel, D.; Xu, Y.; Garcia, A. E. Water Determines the Structure and Dynamics of Proteins Chemical Reviews 2016, 116, 7673.

(3) (a)Ge, A.; Videla, P. E.; Lee, G. L.; Rudshteyn, B.; Song, J.; Kubiak, C. P.; Batista, V. S.; Lian, T. Interfacial Structure and Electric Field Probed by in Situ Electrochemical Vibrational Stark Effect Spectroscopy and Computational Modeling The Journal of Physical Chemistry C 2017, 121, 18674. (b)Sarkar, S.; Maitra, A.; Banerjee, S.; Thoi, V. S.; Dawlaty, J. M. Electric Fields at Metal-Surfactant Interfaces: A Combined Vibrational Spectroscopy and Capacitance Study The Journal of Physical Chemistry B 2020, 124, 1311. (c)Gallo, A.; Farinha, A. S. F.; Dinis, M.; Emwas, A.-H.; Santana, A.; Nielsen, R. J.; Goddard, W. A.; Mishra, H. The chemical reactions in electrosprays of water do not always correspond to those at the pristine air-water interface Chemical Science 2019, 10, 2566. (d)Ruiz-Lopez, M. F.; Francisco, J. S.; Martins-Costa, M. T. C.; Anglada, J. M. Molecular reactions at aqueous interfaces Nature Reviews Chemistry 2020, 4, 459. (e)Chen, L.; Feng, X. Enhanced catalytic reaction at an air-liquid-solid triphase interface Chemical Science 2020, 11, 3124. (f)Barroo, C.; Wang, Z.-J.; Schlögl, R.; Willinger, M.-G. Imaging the dynamics of catalysed surface reactions by in situ scanning electron microscopy Nature Catalysis 2020, 3, 30. (g)Ishizuka, S.; Hama, T.; Enami, S. AcidCatalyzed Oligomerization at the Air-Water Interface Modified by Competitive Adsorption of Surfactants The Journal of Physical Chemistry C 2019, 123, 21662. (h)Yan, Z.; Zhu, L.; Li, Y. C.; Wycisk, R. J.; Pintauro, P. N.; Hickner, M. A.; Mallouk, T. E. The balance of electric field and interfacial catalysis in promoting water dissociation in bipolar membranes Energy \& Environmental Science 2018, 11, 2235.

(4) (a)Kreuzer, H. J. Physics and chemistry in high electric fields Surface and Interface Analysis 2004, 36, 372. (b)Siebert, T.; Guchhait, B.; Liu, Y.; Fingerhut, B. P.; Elsaesser, T. Range, Magnitude, and Ultrafast Dynamics of Electric Fields at the Hydrated DNA Surface The Journal of Physical Chemistry Letters 2016, 7, 3131. (c)Lei, Y. K.; Zhang, J.; Zhang, Z.; Gao, 
Y. Q. Dynamic Electric Field Complicates Chemical Reactions in Solutions The Journal of Physical Chemistry Letters 2019, 10, 2991. (d)Huang, X.; Tang, C.; Li, J.; Chen, L.-C.; Zheng, J.; Zhang, P.; Le, J.; Li, R.; Li, X.; Liu, J.; Yang, Y.; Shi, J.; Chen, Z.; Bai, M.; Zhang, H.-L.; Xia, H.; Cheng, J.; Tian, Z.-Q.; Hong, W. Electric field-induced selective catalysis of single-molecule reaction Science Advances 2019, 5, eaaw3072.

(5) Yan, E. C. Y.; Liu, Y.; Eisenthal, K. B. New Method for Determination of Surface Potential of Microscopic Particles by Second Harmonic Generation The Journal of Physical Chemistry $B$ 1998, 102, 6331 .

(6) (a)Ong, S.; Zhao, X.; Eisenthal, K. B. Polarization of water molecules at a charged interface: second harmonic studies of the silica/water interface Chemical Physics Letters 1992, 191, 327. (b)Zhang, C.; Sprik, M. Electromechanics of the liquid water vapour interface Physical Chemistry Chemical Physics 2020, 22, 10676.

(7) Shaik, S.; Danovich, D.; Joy, J.; Wang, Z.; Stuyver, T. Electric-Field Mediated Chemistry: Uncovering and Exploiting the Potential of (Oriented) Electric Fields to Exert Chemical Catalysis and Reaction Control Journal of the American Chemical Society 2020, 142, 12551.

(8) (a)Goates, S. R.; Schofield, D. A.; Bain, C. D. A Study of Nonionic Surfactants at the Air-Water Interface by SumFrequency Spectroscopy and Ellipsometry Langmuir 1999, 15, 1400. (b)Mafi, A.; Hu, D.; Chou, K. C. Interactions of water with the nonionic surfactant polyoxyethylene glycol alkyl ethers studied by phase-sensitive sum frequency generation and molecular dynamics simulation Surface Science 2016, 648, 366. (c)Shang, B. Z.; Wang, Z.; Larson, R. G. Effect of Headgroup Size, Charge, and Solvent Structure on Polymer-Micelle Interactions, Studied by Molecular Dynamics Simulations The Journal of Physical Chemistry B 2009, 113, 15170. (d)Lu, J. R.; Thomas, R. K.; Penfold, J. Surfactant layers at the air/water interface: structure and composition Advances in Colloid and Interface Science 2000, 84, 143. (e)Lu, J. R.; Su, T. J.; Li, Z. X.; Thomas, R. K.; Staples, E. J.; Tucker, I.; Penfold, J. Structure of Monolayers of Monododecyl Dodecaethylene Glycol at the Air-Water Interface Studied by Neutron Reflection The Journal of Physical Chemistry B 1997, 101, 10332. (f)LucassenReynders, E. H.; Cagna, A.; Lucassen, J. Gibbs elasticity, surface dilational modulus and diffusional relaxation in nonionic surfactant monolayers Colloids and Surfaces A: Physicochemical and Engineering Aspects 2001, 186, 63. (g)Tyrode, E.; Johnson, C. M.; Rutland, M. W.; Claesson, P. M. Structure and Hydration of Poly(ethylene oxide) Surfactants at the Air/Liquid Interface. A Vibrational Sum Frequency Spectroscopy Study The Journal of Physical Chemistry C 2007, 111, 11642. (h)Falciani, G.; Franklin, R.; Cagna, A.; Sen, I.; Hassanali, A.; Chiavazzo, E. A multi-scale perspective of gas transport through soap-film membranes Molecular Systems Design \& Engineering 2020, 5, 911. (i)Nikas, Y. J.; Puvvada, S.; Blankschtein, D. Surface tensions of aqueous nonionic surfactant mixtures Langmuir 1992, 8, 2680. (j)Stubenrauch, C.; Claesson, P. M.; Rutland, M.; Manev, E.; Johansson, I.; Pedersen, J. S.; Langevin, D.; Blunk, D.; Bain, C. D. Mixtures of n-dodecyl- $\beta$-d-maltoside and hexaoxyethylene dodecyl ether - Surface properties, bulk properties, foam films, and foams Advances in Colloid and Interface Science 2010, 155, 5. (k)Shi, L.; Tummala, N. R.; Striolo, A. C12E6 and SDS Surfactants Simulated at the Vacuum-Water Interface Langmuir 2010, 26, 5462.

(9) (a)Shen, Y. R. Phase-Sensitive Sum-Frequency Spectroscopy Annual Review of Physical Chemistry 2013, 64, 129. (b)Eisenthal, K. B. Liquid interfaces Accounts of Chemical
Research 1993, 26, 636. (c)Bredenbeck, J.; Ghosh, A.; Nienhuys, H.-K.; Bonn, M. Interface-Specific Ultrafast Two-Dimensional Vibrational Spectroscopy Accounts of Chemical Research 2009, 42, 1332. (d)Carter, J. A.; Wang, Z.; Dlott, D. D. Ultrafast Nonlinear Coherent Vibrational Sum-Frequency Spectroscopy Methods To Study Thermal Conductance of Molecules at Interfaces Accounts of Chemical Research 2009, 42, 1343. (e)Ge, A.; Rudshteyn, B.; Videla, P. E.; Miller, C. J.; Kubiak, C. P.; Batista, V. S.; Lian, T. Heterogenized Molecular Catalysts: Vibrational Sum-Frequency Spectroscopic, Electrochemical, and Theoretical Investigations Accounts of Chemical Research 2019 , 52, 1289. (f)Shah, S. A.; Baldelli, S. Chemical Imaging of Surfaces with Sum Frequency Generation Vibrational Spectroscopy Accounts of Chemical Research 2020, 53, 1139. (g)Wang, H.-F.; Gan, W.; Lu, R.; Rao, Y.; Wu, B.-H. Quantitative spectral and orientational analysis in surface sum frequency generation vibrational spectroscopy (SFG-VS) International Reviews in Physical Chemistry 2005, 24, 191. (h)Wang, H.-F.; Velarde, L.; Gan, W.; Fu, L. Quantitative SumFrequency Generation Vibrational Spectroscopy of Molecular Surfaces and Interfaces: Lineshape, Polarization, and Orientation Annual Review of Physical Chemistry 2015, 66, 189.

(10) Stiopkin, I. V.; Jayathilake, H. D.; Bordenyuk, A. N.; Benderskii, A. V. Heterodyne-Detected Vibrational Sum Frequency Generation Spectroscopy Journal of the American Chemical Society 2008, 130, 2271.

(11) (a)Strazdaite, S.; Versluis, J.; Backus, E. H. G.; Bakker, H. J. Enhanced ordering of water at hydrophobic surfaces The Journal of Chemical Physics 2014, 140, 054711. (b)Strazdaite, S.; Versluis, J.; Bakker, H. J. Water orientation at hydrophobic interfaces The Journal of Chemical Physics 2015, 143, 084708.

(12) Lu, J. R.; Li, Z. X.; Thomas, R. K.; Staples, E. J.; Tucker, I.; Penfold, J. Neutron reflection from a layer of monododecyl hexaethylene glycol adsorbed at the air-liquid interface: the configuration of the ethylene glycol chain The Journal of Physical Chemistry 1993, 97, 8012.

(13) Abraham, M. J.; Murtola, T.; Schulz, R.; Páll, S.; Smith, J. C.; Hess, B.; Lindahl, E. GROMACS: High performance molecular simulations through multi-level parallelism from laptops to supercomputers SoftwareX 2015, 1-2, 19.

(14) (a)Jorgensen, W. L.; Maxwell, D. S.; Tirado-Rives, J. Development and Testing of the OPLS All-Atom Force Field on Conformational Energetics and Properties of Organic Liquids Journal of the American Chemical Society 1996, 118, 11225. (b)Siu, S. W. I.; Pluhackova, K.; Böckmann, R. A. Optimization of the OPLS-AA Force Field for Long Hydrocarbons Journal of Chemical Theory and Computation 2012, 8, 1459. (c)AbdelAzeim, S. Revisiting OPLS-AA Force Field for the Simulation of Anionic Surfactants in Concentrated Electrolyte Solutions Journal of Chemical Theory and Computation 2020, 16, 1136. (15) Berendsen, H. J. C.; Postma, J. P. M.; van Gunsteren, W. F.; Hermans, J. In Intermolecular Forces: Proceedings of the Fourteenth Jerusalem Symposium on Quantum Chemistry and Biochemistry Held in Jerusalem, Israel, April 13-16, 1981; Pullman, B., Ed.; Springer Netherlands: Dordrecht, 1981, p 331. (16) Zhang, H.; Singer, S. J. Analysis of the Subcritical Carbon Dioxide-Water Interface The Journal of Physical Chemistry A 2011, 115, 6285 .

(17) Zhang, H.; Yin, C.; Jiang, Y.; van der Spoel, D. Force Field Benchmark of Amino Acids: I. Hydration and Diffusion in Different Water Models Journal of Chemical Information and Modeling 2018, 58, 1037. 
(18) Darden, T.; York, D.; Pedersen, L. Particle mesh Ewald: An $\mathrm{N} \cdot \log (\mathrm{N})$ method for Ewald sums in large systems The Journal of Chemical Physics 1993, 98, 10089.

(19) Hess, B.; Bekker, H.; Berendsen, H. J. C.; Fraaije, J. G. E. M. LINCS: A linear constraint solver for molecular simulations Journal of Computational Chemistry 1997, 18, 1463.

(20) Evans, D. J.; Holian, B. L. The Nose-Hoover thermostat The Journal of Chemical Physics 1985, 83, 4069.

(21) (a)Giberti, F.; Hassanali, A. A. The excess proton at the airwater interface: The role of instantaneous liquid interfaces The Journal of Chemical Physics 2017, 146, 244703. (b)Gasparotto, P.; Hassanali, A. A.; Ceriotti, M. Probing Defects and Correlations in the Hydrogen-Bond Network of ab Initio Water Journal of Chemical Theory and Computation 2016, 12, 1953.

(22) Luzar, A.; Chandler, D. Hydrogen-bond kinetics in liquid water Nature 1996, 379, 55.

(23) (a)Reiss, K.; Morzan, U. N.; Grigas, A. T.; Batista, V. S. Water Network Dynamics Next to the Oxygen-Evolving Complex of Photosystem II Inorganics 2019, 7. (b)Negre, C. F. A.; Morzan, U. N.; Hendrickson, H. P.; Pal, R.; Lisi, G. P.; Loria, J. P.; Rivalta, I.; Ho, J.; Batista, V. S. Eigenvector centrality for characterization of protein allosteric pathways Proceedings of the National Academy of Sciences 2018, 115, E12201. (c)Newman, M. E. J. Modularity and community structure in networks Proceedings of the National Academy of Sciences 2006, 103, 8577.

(24) Lange, O. F.; Grubmüller, H. Generalized correlation for biomolecular dynamics Proteins: Structure, Function, and Bioinformatics 2006, 62, 1053.

(25) Nihonyanagi, S.; Ishiyama, T.; Lee, T.-k.; Yamaguchi, S.; Bonn, M.; Morita, A.; Tahara, T. Unified Molecular View of the Air/Water Interface Based on Experimental and Theoretical $\chi(2)$ Spectra of an Isotopically Diluted Water Surface Journal of the American Chemical Society 2011, 133, 16875.

(26) Wijaya, E. C.; Separovic, F.; Drummond, C. J.; Greaves, T. L. Micelle formation of a non-ionic surfactant in non-aqueous molecular solvents and protic ionic liquids (PILs) Physical Chemistry Chemical Physics 2016, 18, 24377.

(27) Tyrode, E.; Johnson, C. M.; Kumpulainen, A.; Rutland, M. W.; Claesson, P. M. Hydration State of Nonionic Surfactant Monolayers at the Liquid/Vapor Interface: Structure Determination by Vibrational Sum Frequency Spectroscopy Journal of the American Chemical Society 2005, 127, 16848.

(28) Gonella, G.; Lütgebaucks, C.; de Beer, A. G. F.; Roke, S. Second Harmonic and Sum-Frequency Generation from Aqueous
Interfaces Is Modulated by Interference The Journal of Physical Chemistry C 2016, 120, 9165.

(29) (a)García Rey, N.; Weißenborn, E.; Schulze-Zachau, F.; Gochev, G.; Braunschweig, B. Quantifying Double-Layer Potentials at Liquid-Gas Interfaces from Vibrational SumFrequency Generation The Journal of Physical Chemistry $C$ 2019, 123, 1279. (b)Dreier, L. B.; Nagata, Y.; Lutz, H.; Gonella, G.; Hunger, J.; Backus, E. H. G.; Bonn, M. Saturation of chargeinduced water alignment at model membrane surfaces Science Advances 2018, 4, eaap7415. (c)Jungwirth, P.; Tobias, D. J. Specific Ion Effects at the Air/Water Interface Chemical Reviews 2006, 106, 1259.

(30) Poli, E.; Jong, K. H.; Hassanali, A. Charge transfer as a ubiquitous mechanism in determining the negative charge at hydrophobic interfaces Nature Communications 2020, 11, 901.

(31) Butt, H. J.; Graf, K.; Kappl, M. Physics and Chemistry of Interfaces; Wiley, 2013.

(32) Nakahara, H.; Shibata, O.; Rusdi, M.; Moroi, Y. Examination of Surface Adsorption of Soluble Surfactants by Surface Potential Measurement at the Air/Solution Interface The Journal of Physical Chemistry C 2008, 112, 6398.

(33) Che, F.; Gray, J. T.; Ha, S.; Kruse, N.; Scott, S. L.; McEwen, J.-S. Elucidating the Roles of Electric Fields in Catalysis: A Perspective ACS Catalysis 2018, 8, 5153.

(34) (a)Nakahara, H.; Shibata, O.; Moroi, Y. Examination of Surface Adsorption of Cetyltrimethylammonium Bromide and Sodium Dodecyl Sulfate The Journal of Physical Chemistry B 2011, 115, 9077. (b)Fernández Leyes, M.; Gimenez Reyes, S.; Cuenca, E.; Sánchez Morales, J. F.; Ritacco, H. Adsorption Kinetics of a Cationic Surfactant Bearing a Two-Charged Head at the Air-Water Interface Coatings 2020, 10.

(35) (a)Ahmadabadi, I.; Esfandiar, A.; Hassanali, A.; Ejtehadi, M. R. Structural and dynamical fingerprints of the anomalous dielectric properties of water under confinement Physical Review Materials 2021, 5, 024008. (b)Schlaich, A.; Knapp, E. W.; Netz, R. R. Water Dielectric Effects in Planar Confinement Physical Review Letters 2016, 117, 048001. (c)Zhu, H.; Yang, F.; Zhu, Y.; Li, A.; He, W.; Huang, J.; Li, G. Investigation of dielectric constants of water in a nano-confined pore RSC Advances 2020, 10, 8628. (d)Senapati, S.; Chandra, A. Dielectric Constant of Water Confined in a Nanocavity The Journal of Physical Chemistry B 2001, 105, 5106. (e)Fumagalli, L.; Esfandiar, A.; Fabregas, R.; Hu, S.; Ares, P.; Janardanan, A.; Yang, Q.; Radha, B.; Taniguchi, T.; Watanabe, K.; Gomila, G.; Novoselov, K. S.; Geim, A. K. Anomalously low dielectric constant of confined water Science 2018, 360, 1339.

SYNOPSIS TOC (Word Style "SN_Synopsis_TOC"). If you are submitting your paper to a journal that requires a synopsis graphic and/or synopsis paragraph, see the Instructions for Authors on the journal's homepage for a description of what needs to be provided and for the size requirements of the artwork.

To format double-column figures, schemes, charts, and tables, use the following instructions:

Place the insertion point where you want to change the number of columns

From the Insert menu, choose Break

Under Sections, choose Continuous

Make sure the insertion point is in the new section. From the Format menu, choose Columns

In the Number of Columns box, type 1

Choose the OK button 
Now your page is set up so that figures, schemes, charts, and tables can span two columns. These must appear at the top of the page. Be sure to add another section break after the table and change it back to two columns with a spacing of $0.33 \mathrm{in}$.

\section{Table 1. Example of a Double-Column Table}

\begin{tabular}{|l|l|l|l|l|l|l|l|}
\hline Column 1 & Column 2 & Column 3 & Column 4 & Column 5 & Column 6 & Column 7 & Column 8 \\
\hline & & & & & & & \\
\hline
\end{tabular}

Authors are required to submit a graphic entry for the Table of Contents (TOC) that, in conjunction with the manuscript title, should give the reader a representative idea of one of the following: A key structure, reaction, equation, concept, or theorem, etc., that is discussed in the manuscript. Consult the journal's Instructions for Authors for TOC graphic specifications.

Insert Table of Contents artwork here 